\title{
THE UNIQUE SUPPORT PATH FOR METASTATIC BREAST CANCER PATIENTS
}

Elana Plotkin ${ }^{1}$, Marianne Gandee ${ }^{2}$, Lorna Lucas ${ }^{1}$, Tara Perloff ${ }^{1}$

1 Association of Community Cancer Centers, Provider Education, Rockville, MD, USA 2 Association of Community Cancer Centers, Development, Rockville, MD, USA

\section{INTRODUCTION}

An estimated 150,000 to 250,000 women in the U.S. are currently living with metastatic breast cancer ${ }^{1}$. While breast cancer is a highprofile disease, receiving significant research funding and prevention initiatives, patients with metastatic breast cancer face unique challenges.

Public messaging about a "cure" is so pervasive that people diagnosed with metastatic breast cancer can be stigmatized by the perception that they've failed to take care of themselves.

\section{OBJECTIVES}

ACCC seeks to expand the current breast cancer conversation to address gaps between early and metastatic disease and improve the treatment and management of metastatic breast cancer in the community setting. This includes addressing the communication challenges that cancer care teams face, such as: patient perception that their cancer is curable, patient level of engagement during conversations with providers, providers who may "minimize" the severity of the disease, and lack of awareness of available resources.

\section{METHODS}

ACCC completed an environmental scan, which combined primary and secondary research to identify existing systems and approaches that effectively support patients with metastatic breast cancer, including key documents to help ACCC understand the gaps and recent innovations that exist within patient support for this patient population.

An expert advisory panel was formed, to include the following members: Joanne Buzgalo, Phd, Cancer Support Community, Helen Chew, MD, UC Davis Cancer Center, Teresa Deshields, PhD, FAPOS, Siteman Cancer Center, William Gradishar, MD, FASCO, FACP, Robert H. Lurie Cancer Center, Julie R. Gralow, MD, Seattle Cancer Care Alliance; Amanda Holt, Maury Regional Medical Center, Shirley Mertz, Metastatic Breast Cancer Alliance; Ruth O'Regan, MD, Carbone Cancer Center, Hester Hill Schnipper, MSW, LICSW, OSW-C, Beth Israel Deaconess Hospital, Lillian Shockney, RN, MAS, Sidney Kimmel Cancer Center.

ACCC selected 3 varying cancer centers and completed in-depth focus groups on their care path including Gibbs Cancer Center, Providence Cancer Center, and Sanford Cancer Center. 16 phone interviews with notable leaders in patient care and support of metastatic breast cancer were conducted.

\section{RESULTS: ACCC Identified 6 Effective Principles}

Principle \#1: Empower the Patient

Principle \#2: Reframe the Conversation

Principle \#3: Reduce Patient Isolation

Principle \#4: Offer Logistical Support at the Cancer Program

Principle \#5: Connect Patients with Support in the Community

Principle \#6: Collaborate in the Interest of Patients

METASTATIC BREAST CANCER COMMUNICATION PROCESS MAP

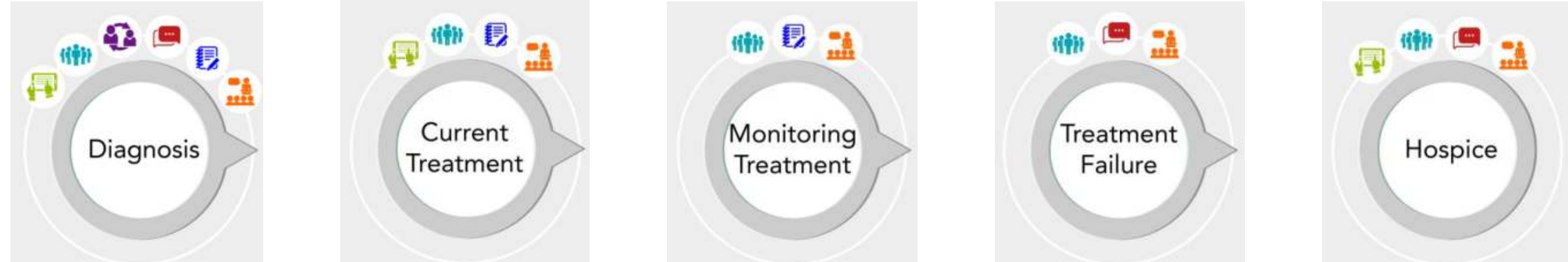

These support principles can be applied throughout the continuum of care, from diagnosis through the end of life. This patient population should be given specialized care to address their unique diagnosis and improve communications with their care team. Each member of the multidisciplinary care team can apply these principles to their workflow. A self-assessment work page is included in this downloadable workbook, so that any team member can identify areas of opportunity to apply these principles to practice.

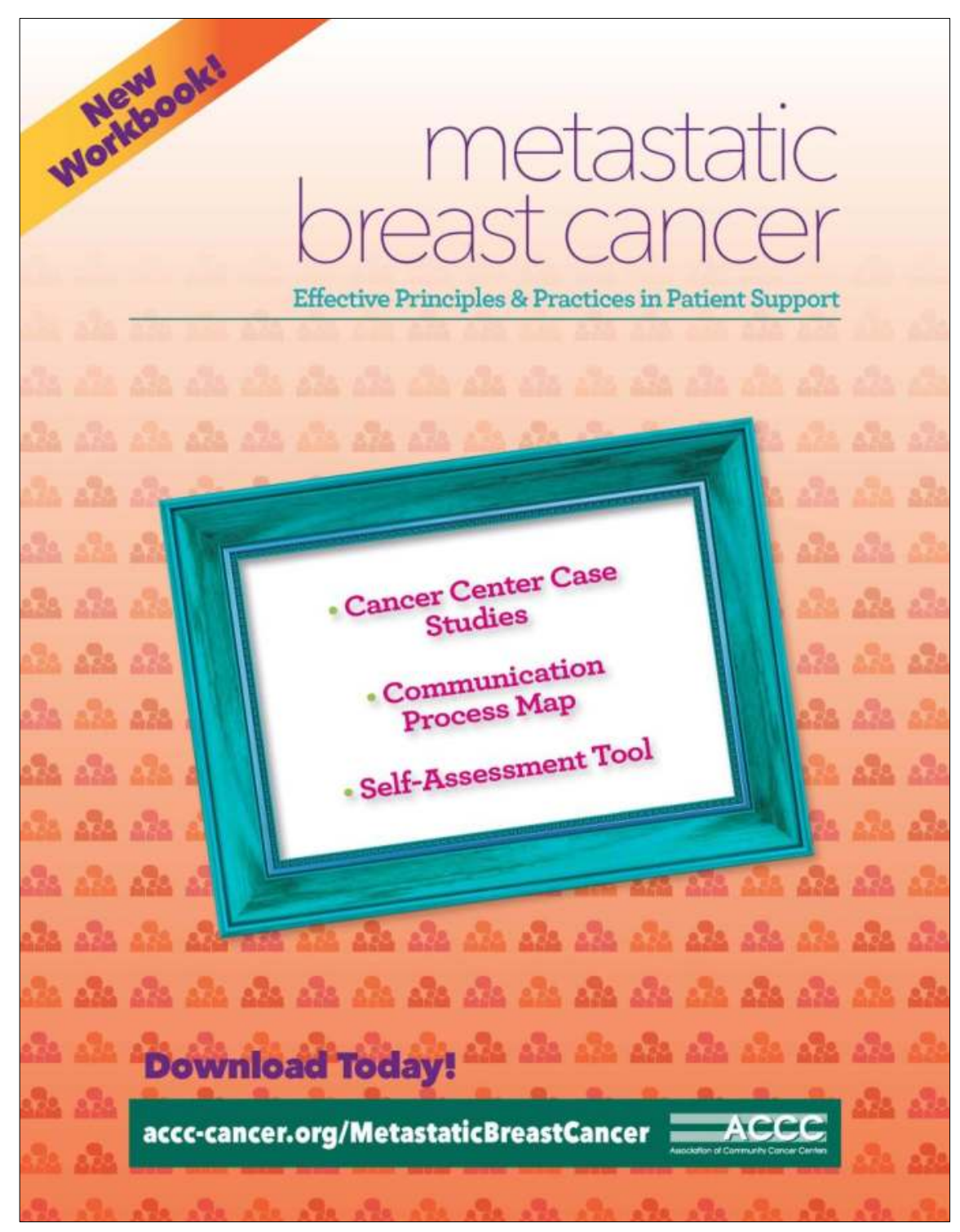

1. Mariotto A, Etzioni R, Hurlbert M, et al. Estimation of the number of women living with metastatic breast cancer in the United States. Cancer Epidemiology Biomarkers Prev. Online May 18, 2017. In print June 1, 2017. DOI: 10.1158/1055-9965.EPI-16-0889.

Continue the conversation about improving care for metastatic breast cancer patients at www.accc-cancer.org/MetastaticBreastCancer, or contact Elana Plotkin, CMP-HC at eplotkin@accc-cancer.org. 